\title{
Merging shuttle reactions and paired electrolysis for reversible vicinal dihalogenations
}

\section{Journal Article}

Author(s):

Dong, Xichang; Roeckl, Johannes L.; Waldvogel, Siegfried R.; Morandi, Bill

Publication date:

2021-01-29

Permanent link:

https://doi.org/10.3929/ethz-b-000466816

Rights / license:

In Copyright - Non-Commercial Use Permitted

Originally published in:

Science 371(6528), https://doi.org/10.1126/science.abf2974

\section{Funding acknowledgement:}

757608 - Shuttle Catalysis for Reversible Molecular Construction (EC)

886102 - Elemental Halogen-Free Reversible Construction and Deconstruction of 1,2- Dihalides via Shuttle Catalysis (EC) 
This document is the Accepted Manuscript version of a Published Work that appeared in final form in Science, copyright (C) American Association for the Advancement of Science after peer review and technical editing by the publisher. To access the final edited and published work see https://doi.org/10.1126/science.abf2974

\title{
Title:
}

\section{Merging shuttle reactions and paired electrolysis for reversible vicinal dihalogenations}

\author{
Authors: Xichang Dong ${ }^{1} \dagger$, Johannes L. Röckl ${ }^{1,2} \dagger$, Siegfried R. Waldvogel ${ }^{2 *} \&$ Bill Morandi $^{1 *}$
}

\begin{abstract}
Affiliations:
${ }^{1}$ Laboratory of Organic Chemistry, Department of Chemistry and Applied Biosciences, ETH

Zürich, Zürich, Switzerland.

${ }^{2}$ Department of Chemistry, Johannes Gutenberg University Mainz, Germany.

*Corresponding author. Email: bill.morandi@org.chem.ethz.ch; waldvogel@uni-mainz.de.

$\uparrow$ These authors contributed equally to this work.
\end{abstract}




\begin{abstract}
:
Vicinal dibromides and dichlorides are important commodity chemicals and indispensable synthetic intermediates in modern chemistry that are traditionally synthesized using hazardous elemental $\mathrm{Cl}_{2}$ and $\mathrm{Br}_{2}$. Meanwhile, the environmental persistence of halogenated pollutants necessitates improved approaches to accelerate their remediation. Here, we introduce an electrochemically-assisted shuttle (e-shuttle) paradigm for the facile and scalable interconversion of alkenes and vicinal dihalides, a class of reactions which can be used both to synthesize useful dihalogenated molecules from simple alkenes and to recycle waste material through retro10 dihalogenation. The reaction is demonstrated using 1,2-dibromoethane, as well as 1,1,1,2tetrachloroethane or 1,2-dichloroethane, to respectively dibrominate or dichlorinate a wide range of alkenes in a simple setup with inexpensive graphite electrodes. Conversely, the hexachlorinated persistent pollutant Lindane could be fully dechlorinated to benzene in soil samples using simple alkene acceptors.
\end{abstract}




\section{Main Text:}

Vicinal dibromides and dichlorides have found widespread applications as flame retardants, pestcontrol agents, polymers and pharmaceuticals $(1,2)$. They also serve as versatile synthetic intermediates in organic chemistry due to the inherent reactivity of carbon-halogen bonds $(3,4)$. Despite these attractive features, the preparation of dihalogenated molecules still mainly relies on the use of highly reactive and corrosive halogenating reagents, such as $\mathrm{Cl}_{2}$ and $\mathrm{Br}_{2}$, which are hazardous compounds to transport, store, and handle (4-7). Two general strategies have been commonly used to avoid the direct use of $\mathrm{Cl}_{2}$ and $\mathrm{Br}_{2}$. The first strategy makes use of carrier reagents, such as $\mathrm{Et}_{4} \mathrm{NCl}_{3}$ or pyridinium tribromide, as bench stable surrogates. Despite their increasing stability, they still require the use of $\mathrm{X}_{2}$ reagents for their syntheses, and tend to be unstable and corrosive themselves since they are designed to readily release the corresponding $\mathrm{X}_{2}$ reagents (5-7). The second strategy relies on the in situ generation of the active halogenating species from the reaction between halides and strong oxidants, a feature which can limit the functional group (e.g. alcohols) compatibility of these reactions (5-7). The use of strong oxidants also creates thermodynamic challenges for the development of the reverse reactions, retrodihalogenations, that could be applied to remediation of persistent halogenated pollutants.

Transfer hydrofunctionalization proceeding through a shuttle catalysis (8) paradigm has emerged as a powerful and versatile strategy to reversibly functionalize and defunctionalize organic molecules without employing or releasing hazardous reagents (8-15), such as $\mathrm{HCN}(9)$. However, catalytic and reversible transfer reactions have so far been limited to alkene monofunctionalization (16) reactions which usually involve the transfer of an $\mathrm{HX}$ molecule $(8,15)$. In contrast, the synthetically appealing, simultaneous transfer of two functional groups, in a catalytic reversible 
transfer difunctionalization process, has remained largely elusive, despite the vast synthetic potential of these reactions in organic synthesis. In particular, reactions involving the formal transfer of extremely reactive and corrosive molecules, such as $\mathrm{Cl}_{2}(17,18)$ or $\mathrm{Br}_{2}$, from easier-tohandle, stable bulk chemicals, such as inexpensive 1,2-dichloro- and 1,2-dibromoethane, would be highly desirable because of the widespread synthetic applications of dihalogenated molecules in flame retardants, pesticides, materials and natural products $(1,2,19)$ (Fig. 1A). The inherent reversibility of such a shuttle reaction would further unlock the facile retro-dihalogenations of end-of-life halogenated products for remediation (Fig. 1A).

The challenge in developing transfer difunctionalizations such as transfer dihalogenations originates from the catalytic approach generally employed in shuttle catalysis. Transfer hydrofunctionalizations, such as hydrocyanation (9), rely on the intermediacy of an alkyl-metal complex which readily undergoes fast and reversible $\beta$-hydride elimination, thus triggering the transfer of a hydrogen atom alongside the desired functional group (15) (Fig. 1B). Unfortunately, the ease of $\beta$-hydride elimination makes the selective, competitive elimination of other synthetically useful groups challenging (20). Furthermore, while $\beta$-hydride elimination is a fast and reversible process, the subsequent migratory re-insertion of an alkene into a metal-halogen bond is often kinetically and thermodynamically disfavored due to the high stability of metalhalogen bonds (6). Thus, a mechanistically distinct approach to favor halogen transfer over hydrogen transfer is crucial to unlock this important class of transfer difunctionalization reactions.

Electrosynthesis has recently experienced a renaissance in organic chemistry, as it takes advantage of readily available electrical current as a sustainable and inherently safe redox reagent (21-24). Notable advances have been made in halogenation reactions $(25,26)$, as illustrated by an elegant example of dichlorination from Lin and coworkers (25). However, this reaction, as well as the vast 
majority of other electrochemical reactions, have to be coupled to another sacrificial half reaction, for example proton reduction to form hydrogen, at the counter-electrode $(23,24)$. Besides this limitation, current protocols can often be further limited by the use of complex reaction setups including expensive metal electrodes $(23,24)$. We envisaged that consecutive paired electrolysis (27) involving a domino reduction-oxidation cascade $(28,29)$, a class of ideal yet comparatively rare electrochemical reactions wherein both electrodes are employed in the desired transformation, could provide a path to reversible electrochemically-mediated shuttle reactions ( $e$-shuttle). We surmised that the reversible cleavage of two strong carbon-halogen bonds through a controlled electron transfer process initiated by a simultaneous, simple reduction and oxidation of key intermediates at the anode and cathode, respectively, would unlock this transformation (Fig. 1C/D). In our hypothesis, the single-electron reduction of the dihalide at the cathode releases the $\mathrm{X}^{-}$anion and generates the carbon radical $\mathbf{1}$, which is almost instantly reduced again to generate a carbanion (30). As a central design, the subsequent selective loss of the second $\mathrm{X}^{-}$instead of a hydride breaks the $\mathrm{C}-\mathrm{X}$ bond, releasing the alkene compound. Considering that a halide anion is a much better leaving group than a hydride, the competing undesired $\beta-\mathrm{H}$ elimination, which is often the preferred pathway when alkyltransition metal complexes are involved as intermediates, can be effectively suppressed by this electrochemical approach. The subsequent oxidation of $\mathrm{X}^{-}$at the anode followed by reaction with the alkene delivers the desired product, which closes the cycle by reestablishing the $\mathrm{C}-\mathrm{X}$ bonds in a fully isodesmic process. Precise control of the potential applied on the electrodes and the highly tunable cell voltage would make this strategy modular and versatile with regard to the group transferred. This is an advantage over the organometallic strategy, where each shuttle reaction 
relies on a completely different combination of metal and catalyst requiring independent optimization campaigns (15).

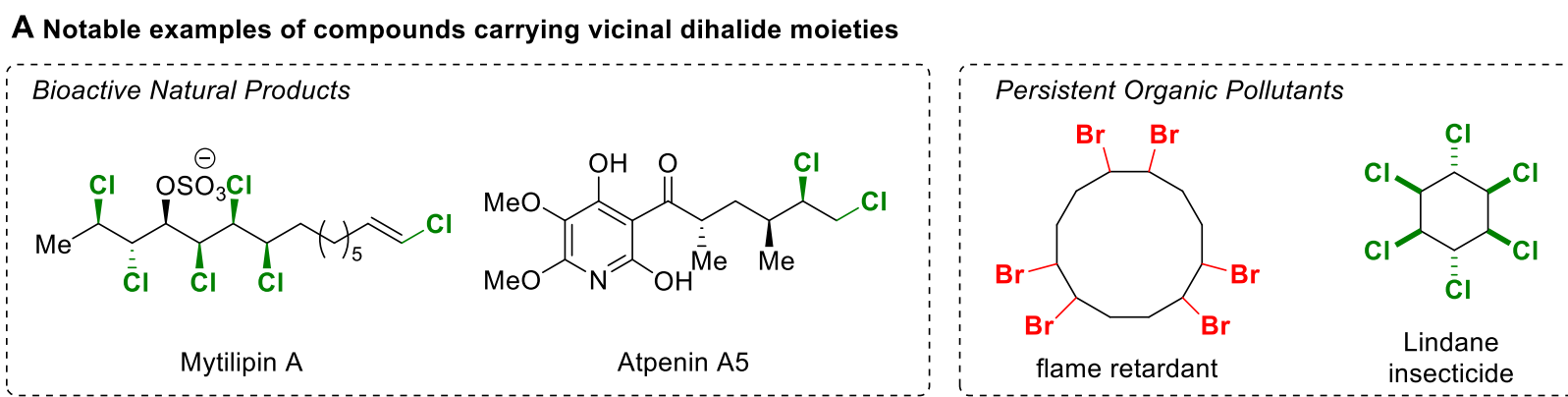

B Transfer hydrofunctionalization and challenges toward the development of transfer difunctionalization

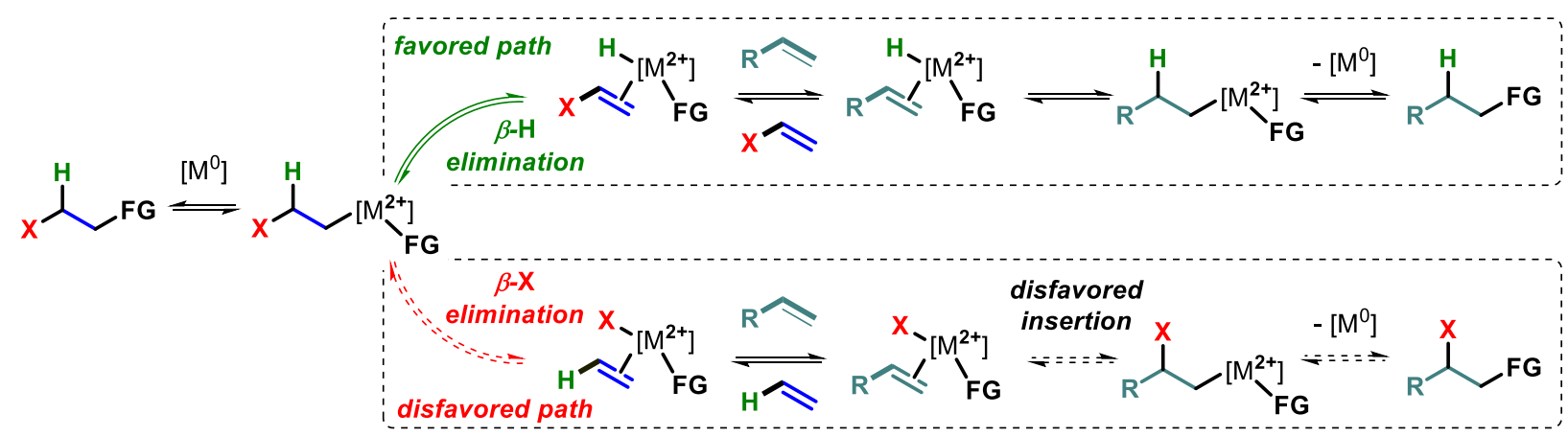

C Electrolysis enabled redox-neutral shuttle reaction (e-shuttle)

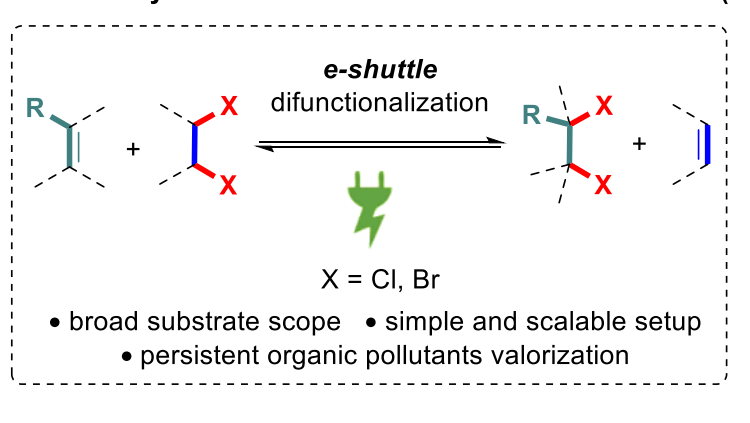

D Reaction design: consecutive paired electrolysis

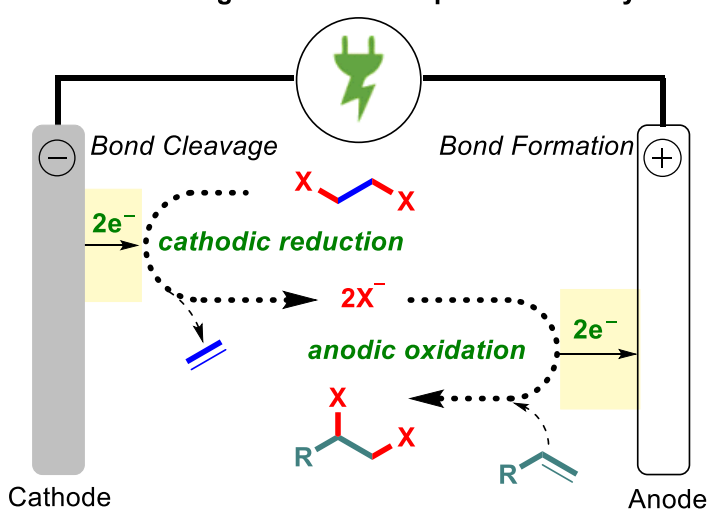

\section{Traditional reagents}

Acutely toxic Highly oxidizing Highly corrosive

$\mathrm{Cl}_{2} \quad \mathrm{Br}_{2}$

Handling, Storage, Transport Handling, Storage, Transport -challenging • expensive • hazardous •easy •inexpensive •safer
Inexpensive surrogates

Lower toxicity Non-oxidizing Non-corrosive

$\mathrm{Cl} \sim \mathrm{Cl} \sim \mathrm{Br}$ 
Fig. 1. Reaction design and challenges of transfer difunctionalization. (A) Notable examples of compounds carrying vicinal dihalide moieties. (B) Transfer hydrofunctionalization and challenges toward the development of transfer difunctionalization. (C) Electrolysis enabled redox-neutral shuttle reaction ( $e$-shuttle). (D) Reaction design: consecutive paired electrolysis. 
At the outset of our investigations, a transfer dibromination was optimized in an undivided cell using inexpensive isostatic graphite as the electrode material under constant current conditions at room temperature, a reaction setup easily accessible to non-specialized laboratories. 1,2-Dibromoethane (DBE) was selected as a formal $\mathrm{Br}_{2}$ donor because it is an inexpensive and stable reagent, produced on a bulk scale, that would release gaseous ethylene as a by-product thus providing a driving force for the shuttle process. Most commercial suppliers offer this reagent at an even lower price (per mol of $\mathrm{Br}_{2}$-equivalents) than $\mathrm{Br} 2$ itself, presumably reflecting the challenges and costs inherent to transporting and storing the volatile and corrosive $\operatorname{Br}_{2}(31)$. Optimal results were obtained with 5 equiv. of 1,2-dibromoethane as the $\mathrm{Br}_{2}$ donor, 1 vol\% $\mathrm{HFIP}$ (1,1,1,3,3,3-hexafluoroisopropanol) as a key additive (32), and 2 equiv. of Et $\mathrm{NBF}_{4}$ as electrolyte in acetonitrile, providing the targeted 1,2-dibromide 2 in $84 \%$ yield (measured by integration of nuclear magnetic resonance spectra) when $3 \mathrm{~F}$ of electricity with respect to 1 -dodecene was applied (Fig. 2). As indicated by cyclic voltammetry (CV) studies, the HFIP facilitates the reduction of the DBE donor and suppresses the undesired and unproductive reductive oligo/polymerization of alkene acceptors at the cathode (see Fig. 2A and Supplementary Fig. S31 and S32 for more details). The supporting electrolyte can be easily crystallized from the reaction mixture to be recycled.

Using this protocol, a broad range of unactivated terminal alkenes $(\mathbf{2}-\mathbf{1 1})$ were readily converted to the corresponding dibromide product in modest to good yields. The reaction conditions were compatible with a large variety of functional groups such as amide (3), ester (4), free carboxylic acid (5), primary alcohol (6), sulfone (7) and bromide (8). Only a small amount of the alcohol group oxidation to aldehyde was observed, confirming the mild nature of the reaction conditions. Activated alkenes, such as styrene (12-15) and vinyl silane (16 and 17), proved to be suitable substrates as well, albeit giving slightly lower yields due to undesired alkene oligomerization. 
While hexa-1,5-diene underwent two-fold 1,2-dibromination to yield the tetra brominated product 18 in acceptable yield, selective mono 1,2-dibromination was observed for several other unconjugated dienes (19 and 20). (E)-4-octene was smoothly dibrominated to produce the mesodibromide $\mathbf{2 1}$ as the single diastereomer. To demonstrate the scalability and robustness of this $e$ shuttle process, the transfer bromination of 1-dodecene was readily scaled-up to a $250 \mathrm{~mL}$ beaker cell from a $10 \mathrm{~mL}$ reaction vial to give $7.58 \mathrm{~g}$ (80\% yield) of product 2 under otherwise identical reaction conditions (Fig. 2C).

Taking advantage of the reversible elimination of a - SR group (33), we could next also develop a transfer bromothiolation of alkenes to prepare 1,2-bromothioether derivatives which are valuable synthetic intermediates usually accessed through multistep synthesis involving highly reactive $\mathrm{R}-\mathrm{SBr}$ reagents $(34,35)$. Several terminal alkenes were successfully converted to the targeted bromothioether product, under otherwise identical conditions, taking 2-bromoethyl phenyl sulfide (5 equiv.) as the $\mathrm{PhS}-\mathrm{Br}$ donor (Fig. 2D). The lower yields arose from competing formation of vicinal disulfides and RS-SR, as well as alkene oligomerization. The ester (23) functional group was compatible. Interestingly, an interrupted shuttle reaction took place when pent-4-en-1-ol and pent-4-enoic acid were employed as the substrates, delivering the cyclic ether (24) or lactone derivatives (25) via subsequent intramolecular nucleophilic attack, demonstrating the method's potential for the development of cascade reactions. 


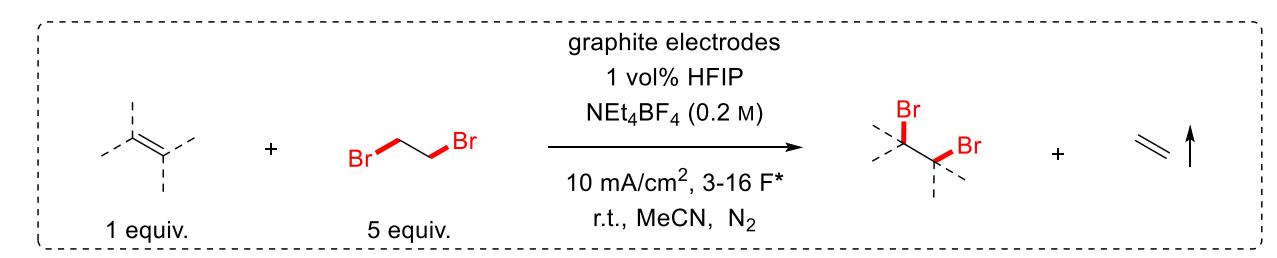

A Cyclic voltammetry studies ${ }^{\dagger}$ : Key role of HFIP
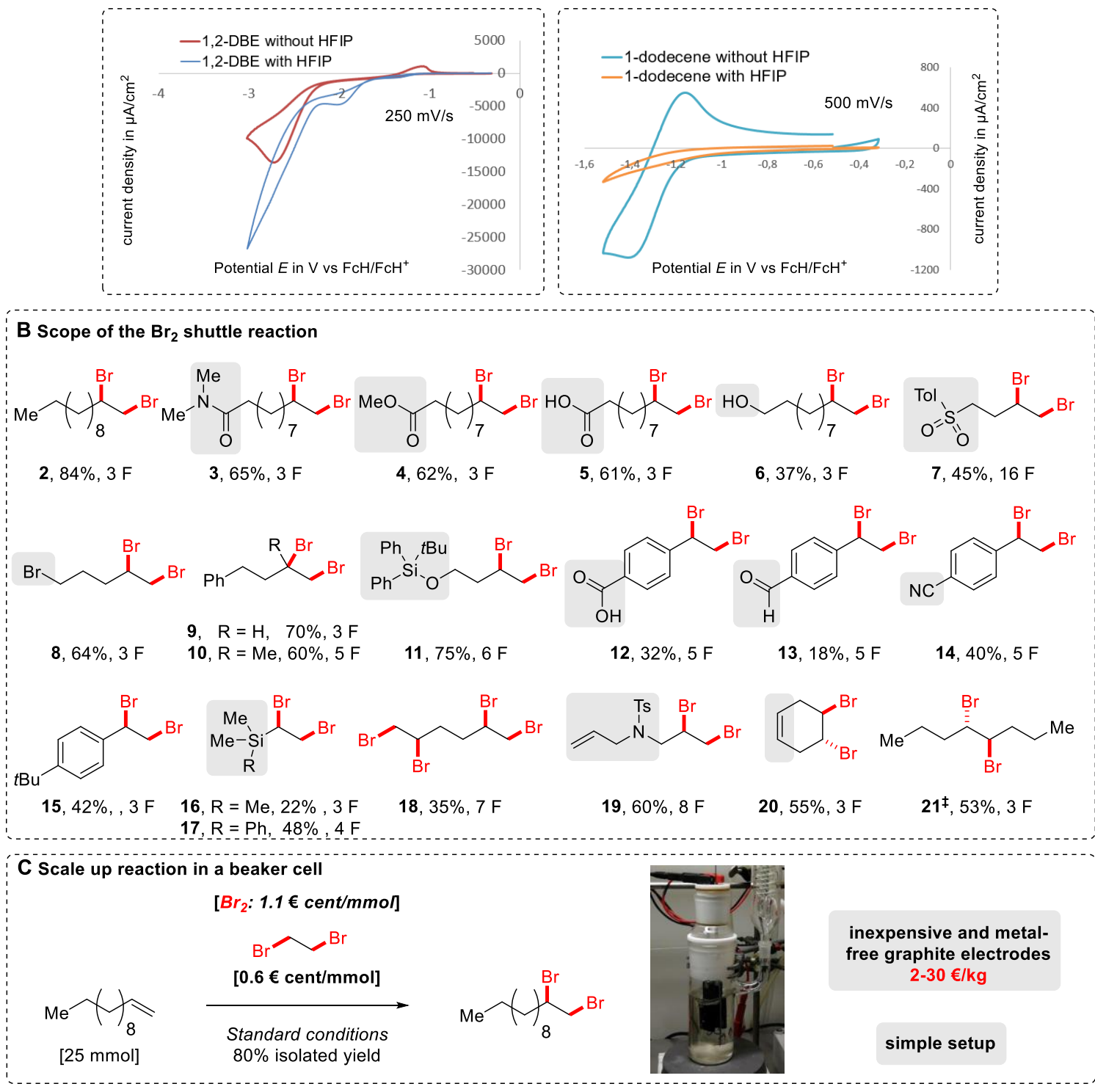

D Scope of the Br-SPh shuttle reaction


Fig. 2. Scope of the $\mathrm{Br}_{2}$ and $\mathrm{Br}-\mathrm{SPh}$ shuttle reactions. All yields are isolated yields of the products. (A) Cyclic voltammetry studies. (B) Scope of the $\mathrm{Br}_{2}$ shuttle reaction. (C) Scale up reaction in a beaker cell. (D) Scope of the $\mathrm{Br}-\mathrm{SPh}$ shuttle reaction. ${ }^{*} 1 \mathrm{~F}$ equals ca. 62 min electrolysis time. $†$ Conditions for $\mathrm{CV}$ studies: A $5 \mathrm{mM}$ solution of 1,2-DBE and 1-dodecene in $\mathrm{MeCN}$ using $\mathrm{NEt}_{4} \mathrm{BF}_{4}(0.2 \mathrm{M})$ as the supporting electrolyte at a graphite electrode with and without 1 vol\% HFIP as the additive. $\sharp(E)$-4-octene used as the starting material. $\S 1 \mathrm{~F}$ equals ca. $31 \mathrm{~min}$ electrolysis time. r.t., room temperature; $\mathrm{FcH}$, ferrocene. r.r., regioisomeric ratio. 
We next explored a transfer dichlorination reaction (Fig. 3). 1,2-Dichloroethane (DCE) was selected as the donor, because it is an inexpensive bulk chemical (20 million ton/year), which is produced as a central intermediate in polyvinylchloride (PVC) production using the excess of $\mathrm{Cl}_{2}$ gas generated during the Chlor-alkali electrolysis process (36). The desired dichloride 28 was obtained in $40 \%$ yield when $5 \mathrm{~mol} \%$ of a $\mathrm{Mn}(\mathrm{II})$ salt (e.g., $\mathrm{MnCl}_{2} \cdot 4 \mathrm{H}_{2} \mathrm{O}$ ) was introduced as a mediator (25) using an otherwise identical electrochemical setup to the dibromination protocol. The yield was further increased to $70 \%$ when DCE (ca. 125 equiv.) was used as the solvent (37). Although this procedure was efficient for a wide set of terminal alkenes (28-34, Fig. 3B), it failed for more challenging 1,1,2-trisubstituted alkene $\mathbf{2 6}$ (Fig. 3A), a feature largely attributable to the undesired 1,2-dechlorinative decomposition of the product 27 and alkene oligomerization of the starting material via cathodic reduction. We reasoned that these two challenges could be smoothly addressed by choosing a more suitable dichloride donor. Based on the known reduction potentials of a large set of simple chlorinated compounds (38), we hypothesized that polychlorinated C2donors, which are more readily reduced, should lead to a more favorable reaction outcome. Experimentally, an excellent correlation between the reduction potential of a series of donors was indeed observed, leading to the identification of 1,1,1,2-tetrachloroethane, a stable, non-corrosive compound, as the reagent of choice, affording the desired dichloride product 27 in $90 \%$ NMR yield (Fig. 3A). Using this procedure, a series of mono-substituted, di-substituted and tri-substituted alkenes participated smoothly in the 1,2-transfer dichlorination reaction, with free alcohol $(\mathbf{2 9}, \mathbf{3 5}$, and 37), ester (30), imide (32), phosphonate (33), sulfone (34), and Ts and Boc protected amine moieties (38 and 39) proving compatible. An internal alkyne (37) was even partially compatible with the reaction conditions, despite the minor formation of unidentified by-products. Various styrene-derived alkenes were converted to the corresponding 1,2-dichlorides in good to excellent yield (42-52), leaving the $\mathrm{Br}, \mathrm{Cl}, \mathrm{CN}, \mathrm{CF}_{3}, \mathrm{CHO}$, and $\mathrm{COOH}$ functional groups untouched. Indene was diastereoselectively transformed into trans-1,2-dichloride 50 (d.r. > 19:1). Interestingly, both $(E)$ - and (Z)-1-phenylpropene were converted to the anti-dichloride $\mathbf{5 1}$ in similarly high diastereoselectivity. The 1,2-dichloride compound 52, bearing a reactive benzylic tertiary $\mathrm{C}-\mathrm{Cl}$ bond, was prepared in good yield from $\alpha$-methylstyrene. Several other activated alkenes, such as the silyl- and ester-substituted alkenes, also proved to be viable substrates to deliver the dichloride products (53-56), in particular, methyl cinnamate was converted to the 1,2-dichloride 56 in an excellent d.r. ratio (> 19:1). To our delight, preliminary experiments showed that this protocol can 
be readily extended to the 1,2-chlorothiolation transfer reaction using the commercially available 2-chloroethyl phenyl sulfide (10 equiv.) as the donor (Fig. 3C). The lower yield can be explained by the undesired formation of RS-SR species as well as substrate oligomerization.

$\mathrm{A} \mathrm{Cl}_{2}$ donor optimization

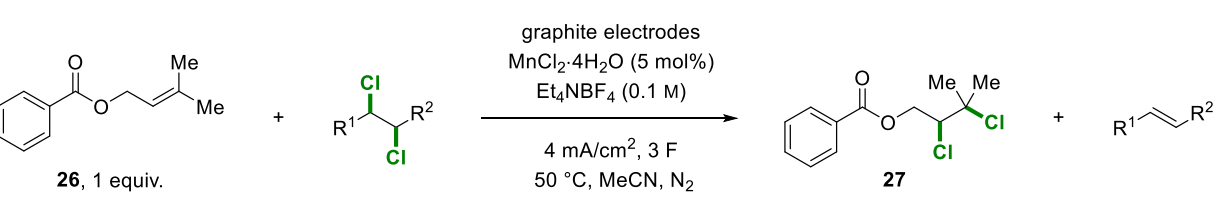

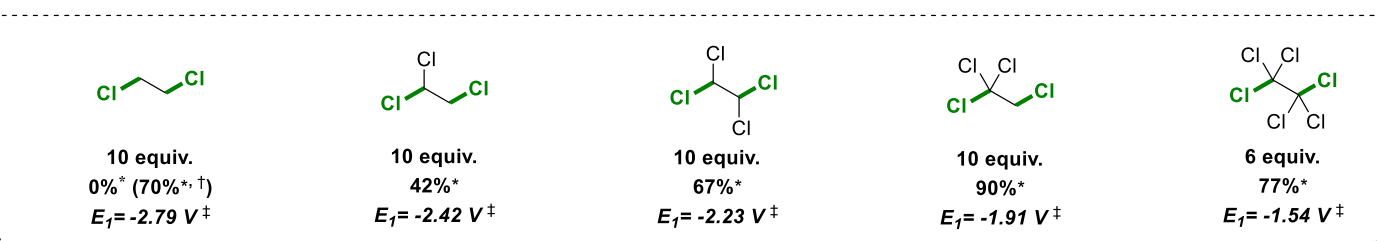

B Scope of the $\mathrm{Cl}_{2}$ shuttle reaction

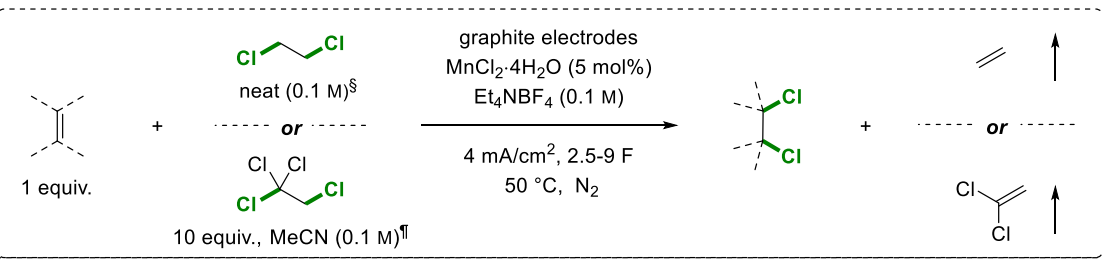

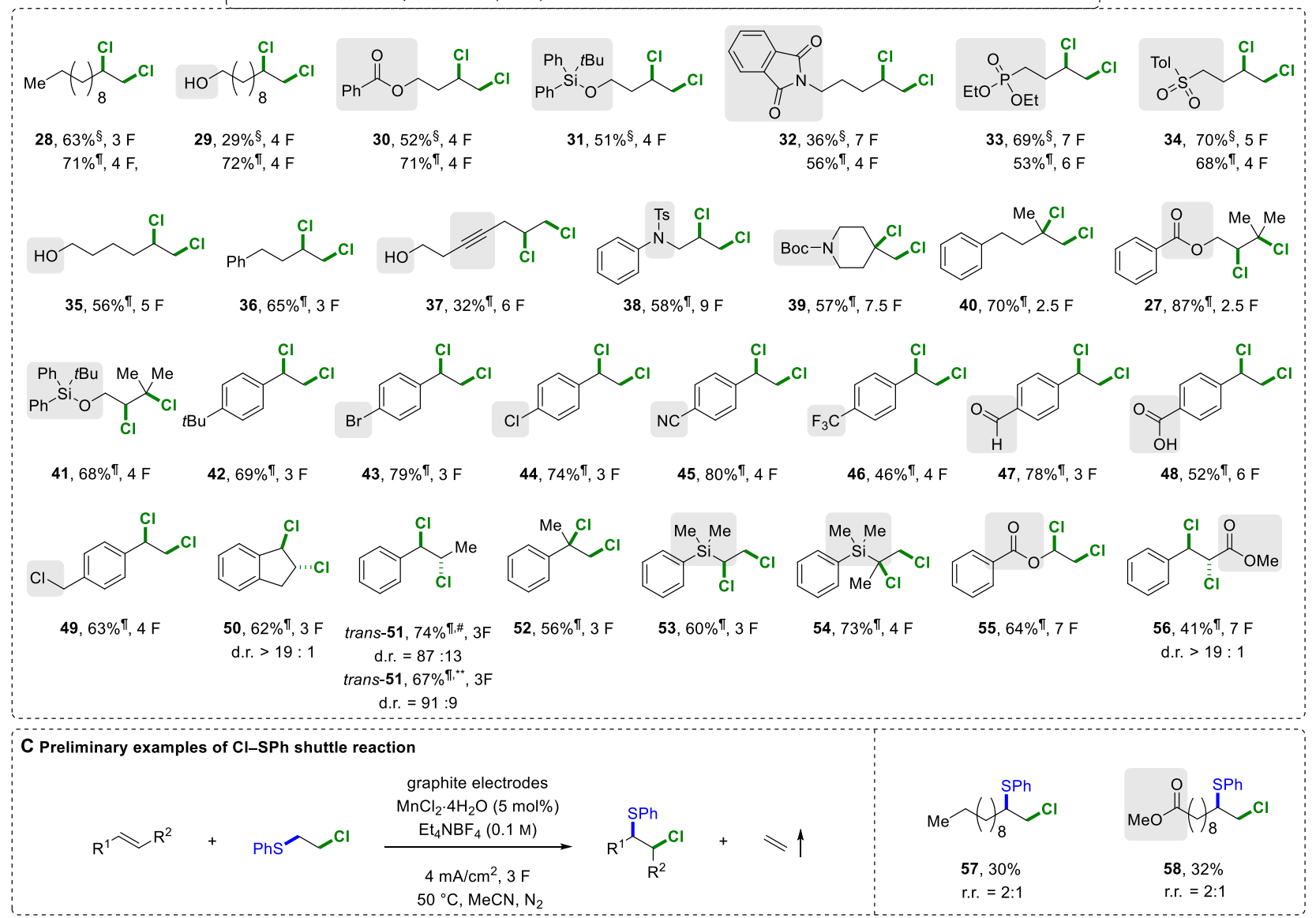


Fig. 3. Scope of the $\mathrm{Cl}_{2}$ and $\mathbf{C l}-\mathrm{SPh}$-shuttle reactions. $1 \mathrm{~F}$ equals ca. 81 min electrolysis time. All yields are isolated yields of the products unless otherwise noted. (A) $\mathrm{Cl}_{2}$ donor optimization. (B) Scope of the $\mathrm{Cl}_{2}$ shuttle reaction. (C) Preliminary examples of $\mathrm{Cl}-\mathrm{SPh}$ shuttle reaction. ${ }^{* 1} \mathrm{H}$ NMR yield with mesitylene as the internal standard. $†$ Neat DCE $(0.1 \mathrm{M})$ as the donor, 1-dodecene (1 equiv.) as the acceptor. $₫$ Redox potential (V vs SCE) measured for the first reduction peak at $0.2 \mathrm{Vs}^{-1}$, polychlororethanes $(2 \mathrm{mM})$ in $\mathrm{DMF}+0.1 \mathrm{M}\left(\mathrm{C}_{3} \mathrm{H}_{7}\right)_{4} \mathrm{NBF}_{4}$ at a glassy carbon electrode according to (38). §Neat DCE $(0.1 \mathrm{M})$ as the donor. I[Reaction performed in $\mathrm{MeCN}(0.1 \mathrm{M})$ with 1,1,1,2tetrachloroethane as the donor. \#(E)-prop-1-en-1-ylbenzene used as the starting material. **(Z)-prop-1-en-1ylbenzene used as the starting material. d.r., diastereomeric ratio; r.r., regioisomeric ratio. 
In contrast to traditional halogenation methods, the inherent reversibility of the $e$-shuttle strategy offers a platform to develop retro-dihalogenation reactions. Given the environmental persistence of several halogenated compounds produced at commodity scale, such as flame retardants and insecticides, $e$-shuttle could facilitate their recycling and valorization through retrodihalogenations, which could ultimately lead to a circular economy for these important chemicals. A notable example is Lindane (gamma-hexachlorocyclohexane), a compound that was once used worldwide as an effective broad-spectrum insecticide in crop protection, which is now classified as a persistent organic pollutant due to its high toxicity and high persistency in the environment (39-42). Global quantities of hexachlorocyclohexane $(\mathrm{HCH})$ wastes still present in the environment range between 4-7 million tons worldwide, highlighting the pressing challenge in finding methods to recycle and remove this compound from contaminated soils (39). We thus questioned whether this waste material, which, among other chemical and biological approaches (40), can only be inefficiently degraded through normal electrochemical recycling methods (4345), could be efficiently retro-dihalogenated using our $e$-shuttle strategy (Fig. 4A). Indeed, Lindane, through three successive retro-dichlorination events, successfully transferred its six chloride atoms to an acceptor alkene to form benzene, the fully dechlorinated by-product of Lindane, alongside a dichlorinated alkane. Five illustrative alkene examples gave excellent yields up to $89 \%$ (with respect to 3.0 equiv. of $\mathrm{Cl}_{2}$-equivalents of Lindane at $>95 \%$ GC yield of benzene, Fig. 4B), demonstrating the generality of this process and the possibility to access a wide variety of potentially useful chemicals. A scale-up experiment ( $87.5 \mathrm{mmol}$ of alkene), further showcased the efficiency of the retro-dichlorination process (Fig. 4C). The exceptional functional group tolerance of our $e$-shuttle strategy made us next question whether we could successfully retrodichlorinate Lindane-contaminated soils through a transfer dichlorination reaction (Fig. 4D). In 
theory, such a process could lead to a new avenue to chemically remediate highly contaminated soils, which are mainly caused by leachates of improper disposal at landfilling or dump sites (39, $40,42)$, through simultaneous synthesis of commercially relevant dichlorinated chemicals. This could provide an alternative to current industrial approaches that focus on the generation of $\mathrm{HCl}$ from chlorinated waste (42).

Artificially contaminated soils are commonly used as models in environmental chemistry to perform proof-of-concept chemical remediation experiments (46). Therefore, to mimic the composition of soils contaminated by high concentration of hexachlorocyclohexane, three soil samples from different locations near our university campus, i.e., roadside, flower field, and farmland, were collected and homogeneously mixed with commercially available Lindane. Remarkably, the $50 \mathrm{w} \%$ Lindane contaminated soil could be used directly in the reaction without any pre-extraction or filtration, delivering both the benzene and dichloride product in excellent yields and high purity (Entry 1-3, Fig. 4D), a result comparable to the experiments using pure Lindane (Entry 5, Fig. 4D). This result shows that our degradation process is compatible with the biological and mineral impurities present in three different soil types. While the exact composition of environmentally-relevant contaminated soils might significantly differ from our samples (39, 41-42), we nevertheless believe that these positive preliminary results, using a variety of soil samples, support the feasibility of this approach. A much lower Lindane-soil ratio of $1 \mathrm{w} \%$, where Lindane was extracted with the reaction solvent prior to the degradation, also afforded good yields for both benzene (76\%) and dichloride (76\%, Entry 4, Fig. 4D). This alternative pre-extraction protocol acts as a further proof-of-concept which might help the design of larger scale remediation processes in which undesired soil contamination with electrolyte and Mn catalyst can be prevented. Interestingly, the large-scale feasibility of an extraction approach has been demonstrated by the 
successful treatment of ca. 70,000 tons of $\mathrm{HCH}$ contaminated soils in the Netherlands in a fullscale soil washing plant, which achieved HCH removal efficiency of more than 99.7\% (42).

Collectively, these preliminary results serve as a proof-of-principle for the direct remediation of Lindane-contaminated soils using $e$-shuttle methodology. 

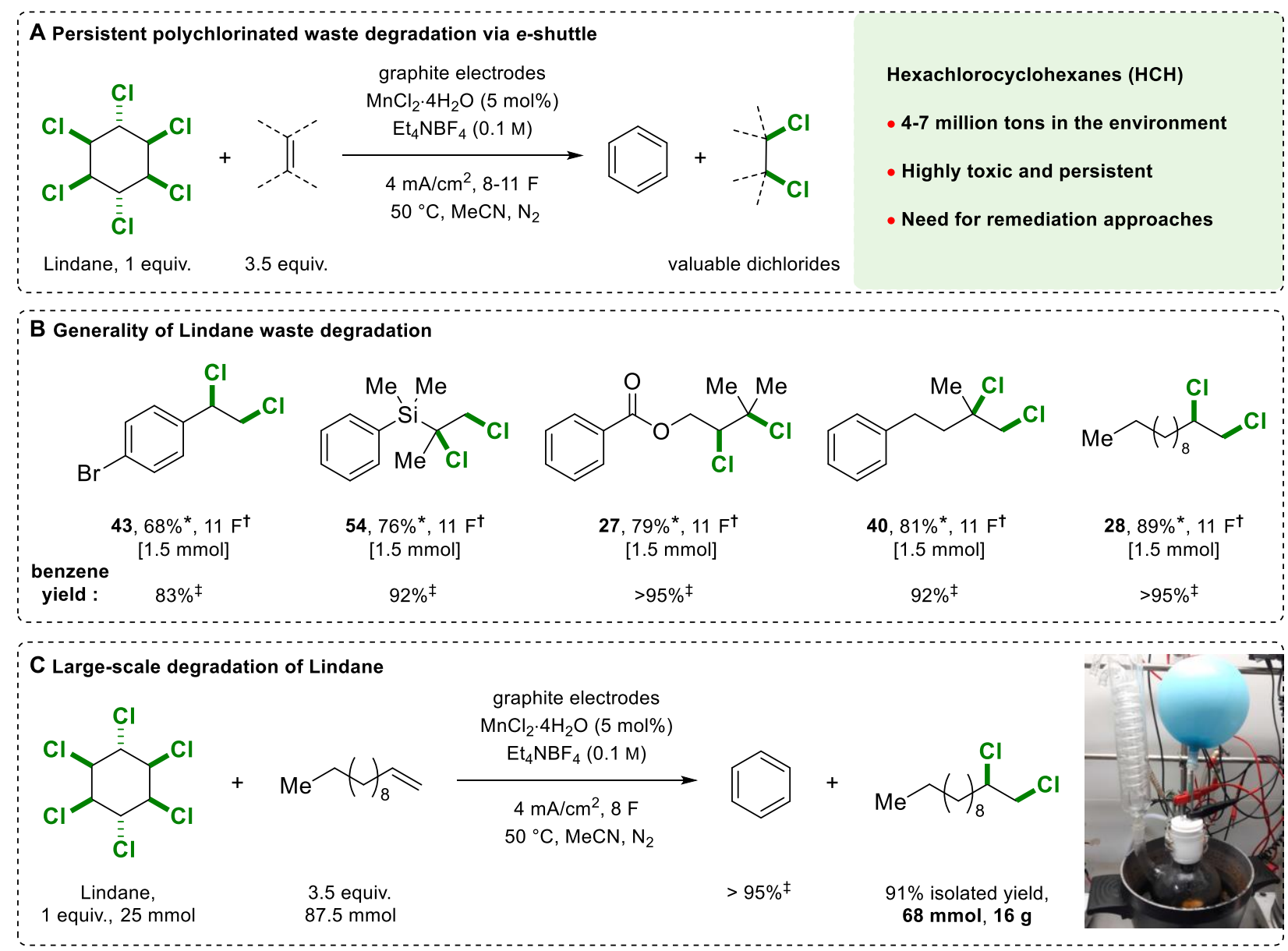

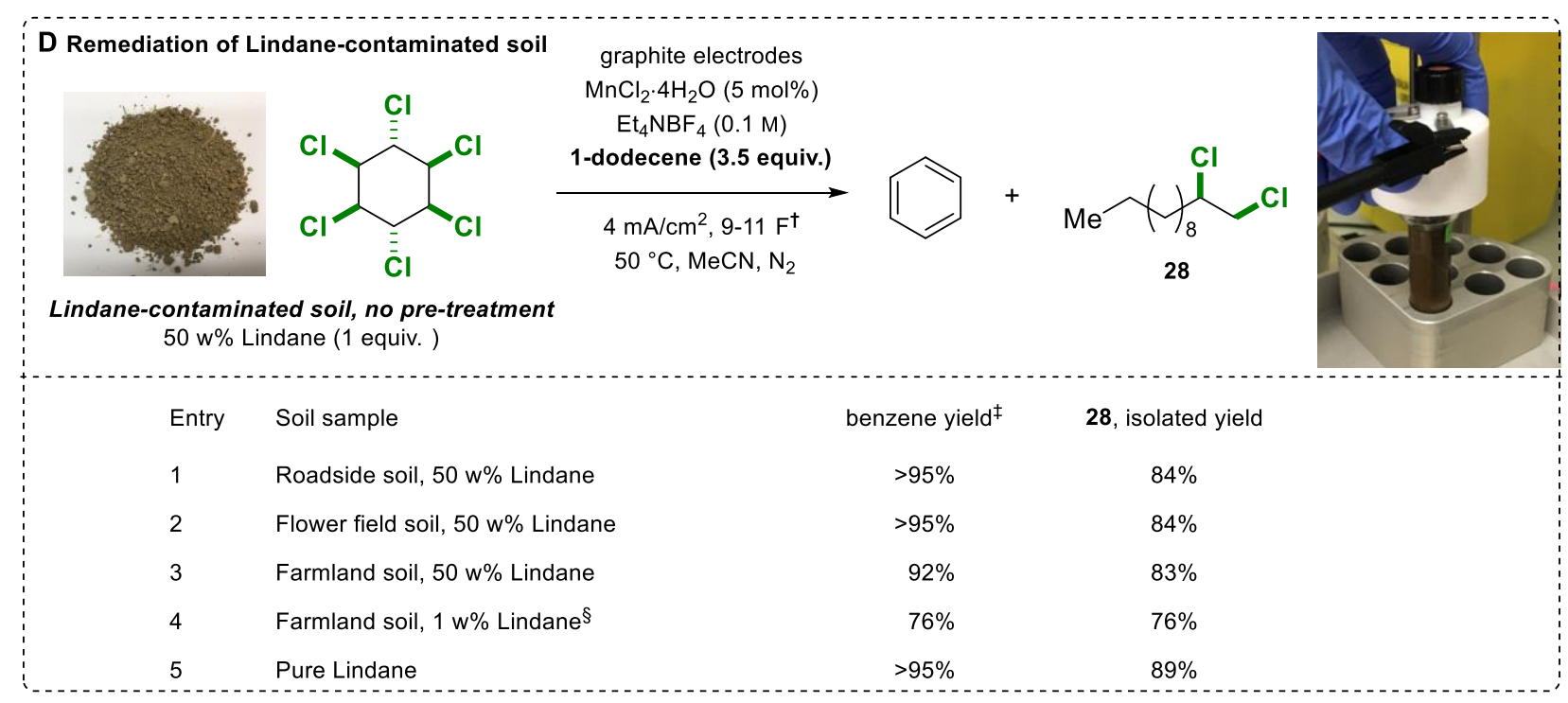

Fig. 4. Application of $\boldsymbol{e}$-shuttle reactions. (A) Persistent polychlorinated waste degradation via $e$-shuttle. (B) Generality of Lindane waste degradation. (C) Large-scale degradation of Lindane. (D) Remediation of Lindane- 
contaminated soil. *Isolated yield. $\dagger 1 \mathrm{~F}$ equals ca. 81 min electrolysis time. $†$ Yield measured by gas chromatography using mesitylene or anisole as the internal standard. §Lindane was extracted with $\mathrm{MeCN}$ before degradation.

\section{References and Notes:}

1. K. L. Kirk, Persistent polyhalogenated compounds: biochemistry, toxicology, medical applications, and associated environmental issues. in Biochemistry of the Elemental Halogens and Inorganic Halides (Springer, 1991), pp. 191-238.

2. M. M. Häggblom, Bossert, I. D. Halogenated organic compounds-a global perspective. in Dehalogenation Microbial Processes and Environmental Applications (Springer, 2004) pp. 3-29.

3. S. Patai, Eds, The Chemistry of The Carbon-Halogen Bond: Part 1 (Wiley, 1973).

4. I. Saikia, A. J. Borah, P. Phukan, Use of bromine and bromo-organic compounds in organic synthesis. Chem. Rev. 116, 6837-7042 (2016).

5. M. Eissen, D. Lenoir, Electrophilic bromination of alkenes: environmental, health and safety aspects of new alternative methods. Chem. Eur. J. 14, 9830-9841 (2008).

6. A. J. Cresswell, S. T.-C. Eey, S. E. Denmark, Catalytic, stereoselective dihalogenation of alkenes: challenges and opportunities. Angew. Chem. Int. Ed. 54, 15642-15682 (2015).

7. W. J. Chung, C. D. Vanderwal, Stereoselective halogenation in natural product synthesis. Angew. Chem. Int. Ed. 55, 4396-4434 (2016).

8. B. N. Bhawal, B. Morandi, Catalytic transfer functionalization through shuttle catalysis. ACS Catal. 6, 7528-7535 (2016).

9. X. Fang, P. Yu, B. Morandi, Catalytic reversible alkene-nitrile interconversion through controllable transfer hydrocyanation. Science 351, 832-836 (2016).

10. X. Fang, B. Cacherat, B. Morandi, CO-and HCl-free synthesis of acid chlorides from unsaturated hydrocarbons via shuttle catalysis. Nat. Chem. 9, 1105-1109 (2017). 
11. S. K. Murphy, J.-W. Park, F. A. Cruz, V. M. Dong, Rh-catalyzed C-C bond cleavage by transfer hydroformylation. Science 347, 56-60 (2015).

12. D. A. Petrone, et al., M. Palladium-catalyzed hydrohalogenation of 1, 6-enynes: hydrogen halide salts and alkyl halides as convenient HX Surrogates. J. Am. Chem. Soc. 139, 35463557 (2017).

13. W. Chen, J. C. Walker, M. Oestreich, Metal-free transfer hydroiodination of C-C multiple bonds. J. Am. Chem. Soc. 141, 1135-1140 (2018).

14. P. Yu, A. Bismuto, B. Morandi, Iridium-catalyzed hydrochlorination and hydrobromination of alkynes by shuttle catalysis. Angew. Chem. Int. Ed. 59, 2904-2910 (2020).

15. B. N. Bhawal, B. Morandi, Catalytic isofunctional reactions - expanding the repertoire of shuttle and metathesis reactions. Angew. Chem. Int. Ed. 58, 10074-10103 (2019).

16. M. Beller, J. Seayad, A. Tillack, H. Jiao, Catalytic Markovnikov and anti-Markovnikov functionalization of alkenes and alkynes: recent developments and trends. Angew. Chem. Int. Ed. 43, 3368-3398 (2004).

17. K. Sakai, K. Sugimoto, S. Shigeizumi, K. Kondo, A new selective dichlorination of C-C double bonds. Tetrahedron Lett. 35, 737-740 (1994).

18. M. L. Ho, A. B. Flynn, W. W. Ogilvie, Single-isomer iodochlorination of alkynes and chlorination of alkenes using tetrabutylammonium iodide and dichloroethane. J. Org. Chem. 72, 977-983 (2007).

19. G. W. Gribble, Naturally occurring organohalogen compounds. Acc. Chem. Res. 31, 141$152(1998)$.

20. R. H. Crabtree, The Organometallic Chemistry of the Transition Metals, (Wiley, ed. 5, 2009). 
21. M. Yan, Y. Kawamata, P. S. Baran, Synthetic organic electrochemical methods since 2000: on the verge of a renaissance. Chem. Rev. 117, 13230-13319 (2017).

22. J. C. Siu, N. Fu, S. Lin, Catalyzing electrosynthesis: a homogeneous electrocatalytic approach to reaction discovery. Acc. Chem. Res. 53, 547-560 (2020).

23. J. L. Röckl, D. Pollok, R. Franke, S. R. Waldvogel, A decade of electrochemical dehydrogenative C, C-coupling of aryls. Acc. Chem. Res. 53, 45-61 (2020).

24. A. Wiebe, T. Gieshoff, S. Möhle, E. Rodrigo, M. Zirbes, S. R. Waldvogel, Electrifying organic synthesis. Angew. Chem. Int. Ed. 57, 5594-5619 (2018).

25. N. Fu, G. S. Sauer, S. Lin, Electrocatalytic radical dichlorination of alkenes with nucleophilic chlorine sources. J. Am. Chem. Soc. 139, 15548-15553 (2017).

26. Y. Yuan, et al., Electrochemical oxidative clean halogenation using $\mathrm{HX} / \mathrm{NaX}$ with hydrogen evolution. iScience 12, 293-303 (2019).

27. Y. Mo, et al., Microfluidic electrochemistry for single-electron transfer redox-neutral reactions. Science 368, 1352-1357 (2020).

28. M. F. Hartmer, S. R. Waldvogel, Electroorganic synthesis of nitriles via a halogen-free domino oxidation-reduction sequence. Chem. Commun. 51, 16346-16348 (2015).

29. D. Pollok, S. R. Waldvogel, Electro-organic synthesis-a $21^{\text {st }}$ century technique. Chem. Sci. 11, 12386-12400 (2020).

30. J. Casanova, L. Eberson, Electrochemistry of the Carbon-Halogen Bond. in The Chemistry of The Carbon-Halogen Bond: Part 1 (Wiley, 1973), pp. 979-1047.

31. D. M. Hill, "Safety review of bromine-based electrolytes for energy storage applications", Report 1 http://energystorageicl.com/wp-content/uploads/2018/04/DNV-GL-SafetyReview-of-Bromine-Based-Electrolytes-for-Energy-Storage-Applications.pdf (2018). 
32. L. Schulz, S. R. Waldvogel, Solvent control in electro-organic synthesis, Synlett 30, 275286 (2019).

33. S. E. Denmark, W. R. Collins, M. D. Cullen, Observation of direct sulfenium and selenenium group transfer from thiiranium and seleniranium ions to alkenes. J. Am. Chem. Soc. 131, 3490-3492 (2009).

34. E. Schneider, Darstellung und Eigenschaften von Alkylschwefelhalogeniden, Chem. Ber. 84, 911-916 (1951).

35. J. Drabowicz, P. Kiełbasiński, M. Mikołajczyk, Synthesis of sulphenyl halides and sulphenamides. in Sulfenic Acids and Derivatives (Wiley, 1990), pp. 221-292.

36. C. Hoffmann, J. Weigert, E. Esche, J. U. Repke, Towards demand-side management of the chlor-alkali electrolysis: Dynamic, pressure-driven modeling and model validation of the 1, 2-dichloroethane synthesis. Chem. Eng. Sci. 214, 115358 (2020).

37. Y. Liang, F. Lin, Y. Adeli, R. Jin, N. Jiao, Efficient electrocatalysis for the preparation of (hetero)aryl chlorides and vinyl chlorides with 1,2-dichloroethane. Angew. Chem. Int. Ed. 58, 4566-4570 (2019).

38. B. Huang, A. A. Isse, C. Durante, C. Wie, A. Gennaro, Electrocatalytic properties of transition metals toward dichlorination of polychloroethanes. Electrochim. Acta 70, 50-61 (2012).

39. J. Vijgen, B. de Borst, R. Weber, T. Stobiecki, M. Forter, HCH and Lindane contaminated sites: European and global need for a permanent solution for a long-time neglected issue. Environ. Pollut. 248, 696-705 (2019).

40. P. Bhatt, M. S. Kumar, T. Chakrabarti, Fate and degradation of POPhexachlorocyclohexane. Crit. Rev. Environ. Sci. Technol. 39, 655-695 (2009). 
41. K. Walker, D. A. Vallero, R. G. Lewis, Factors influencing the distribution of Lindane and other hexachlorocyclohexanes in the environment. Environ. Sci. Technol. 33, 4373-4378 (1999).

42. M. Vega, D. Romano, E. Uotila. Lindane (persistent organic pollutant) in the EU. Directorate General for Internal Policies. Policy Department C: Citizens' Rights and Constitutional Affairs. Petitions (PETI). PE 571.398 (2016).

43. S. Rondinini, A. Vertova, Electroreduction of halogenated organic compounds. in Electrochemistry for the Environment (Springer, 2010), pp. 279-306.

44. J. P. Merz, B. C. Gamoke, M. P. Foley, K. Raghavachari, D. G. Peters, Electrochemical reduction of $(1 R, 2 r, 3 S, 4 R, 5 r, 6 S)$-hexachlorocyclohexane (Lindane) at carbon cathodes in dimethylformamide. J. Electroanal. Chem. 660, 121-126 (2011).

45. E. T. Martin, C. M. McGuire, M. S. Mubarak, D. G. Peters, Electroreductive remediation of halogenated environmental pollutants. Chem. Rev. 116, 15198-15234 (2016).

46. E. Morill, J. Villaverde, Advanced technologies for the remediation of pesticidecontaminated soils. Sci. Total Environ. 586, 576-597 (2017).

\section{Listed here are references from the supporting materials.}

47. G. R. Fulmer, et al., NMR chemical shifts of trace impurities: common laboratory solvents, organics, and gases in deuterated solvents relevant to the organometallic chemist. Organometallics 29, 2176-2179 (2010).

48. J. Liu, Q. Ren, X. Zhang, H. Gong, Preparation of vinyl arenes by Nickel-catalyzed reductive coupling of aryl halides with vinyl bromides. Angew. Chem. Int. Ed. 55, $15544-15548$ (2016). 
49. A. K. Macharla, R. C. Nappunni, N. Nama, Regio- and stereoselective hydroxybromination and dibromination of olefins using ammonium bromide and oxone ${ }^{\circledR}$. Tetrahedron Lett. 53, $1401-1405$ (2012).

50. S. Lethu, S. Matsuoka, M. Murata, Highly efficient preparation of selectively isotope cluster-labeled long chain fatty acids via two consecutive $\mathrm{Csp}^{3}-\mathrm{Csp}^{3}$ cross-coupling reactions. Org. Lett. 16, 844-847 (2014).

51. J. Long, J. Chen, R. Li, Z. Liu, X. Xiao, J. H. Lin, J. C. Xiao, Ph3 P/I-Promoted dichlorination or dibromination of epoxides with $\mathrm{XCH}_{2} \mathrm{CH}_{2} \mathrm{X}(\mathrm{X}=\mathrm{Cl}$ or Br). Synlett $\mathbf{3 0}$, 181-184 (2019).

52. G. W. Kabalka, K. Yang, N. K. Reddy, C. Narayana, Bromination of alkenes using a mixture of sodium bromide and sodium perborate. Synth. Commun. 28, 925-929 (1998).

53. K. Yubata, H. Matsubara, Atom-economical brominations with tribromide complexes in the presence of oxidants. Tetrahedron Lett. 60, 1001-1004 (2019).

54. X. Xia, P. H. Toy, Rasta resin-triphenylphosphine oxides and their use as recyclable heterogeneous reagent precursors in halogenation reactions. Beilstein J. Org. Chem. 10, 1397-1405 (2014).

55. W. Chen, H. Tao, W. Huang, G. Wang, S. Li, X. Cheng, G. Li, Hantzsch ester as a photosensitizer for the visible-light-induced debromination of vicinal dibromo compounds. Chem. Eur. J. 22, 9546-9550 (2016).

56. A. Chassepot, et al., Chemically detachable polyelectrolyte multilayer platform for cell sheet engineering. Chem. Mater. 24, 930-937 (2012).

57. N. S. Martins, E. E. Alberto, Dibromination of alkenes with $\mathrm{LiBr}$ and $\mathrm{H}_{2} \mathrm{O}_{2}$ under mild conditions. New J. Chem. 42, 161-167 (2018). 
58. W. E. Billups, R. K. Saini, V. A. Litosh, L. B. Alemany, W. K. Wilson, K. B. Wiberg, Thermal rearrangements of spiro [2.4] hepta-1, 4, 6-trienes. J. Org. Chem. 67, 4436-4440 (2002).

59. K. M. Redies, T. Fallon, M. Oestreich, En Route to stable all-Carbon-substituted silylenes: synthesis and reactivity of a bis( $\alpha$-spirocyclopropyl) silylene. Organometallics 33, 3235-3238 (2014).

60. R. Campagne, F. Schäkel, R. Guillot, V. Alezra, C. Kouklovsky, Base-mediated fragmentation of bicyclic dihydro-3, 6-oxazines: transformation of nitroso Diels-Alder cycloadducts. Org. Lett. 20, 1884-1887 (2018).

61. D. Lexa, J. M. Saveant, H. J. Schaefer, S. K. Binh, B. Vering, D. L. Wang, Outer-sphere and inner-sphere processes in reductive elimination. Direct and indirect electrochemical reduction of vicinal dibromoalkanes. J. Am. Chem. Soc. 112, 6162-6177 (1990).

62. L. Benati, P. C. Montevecchi, P. Spagnolo, Synthetic utility of 4'nitrobenzenesulfenanilide in the functionalization of carbon-carbon double and triple bonds: Its use in the bromosulfenylation of alkenes and alkynes. Tetrahedron 49, 5365-5376 (1993).

63. X. Marset, G. Guillena, D. J. Ramón, Deep eutectic solvents as reaction media for the palladium-catalysed $\mathrm{C}-\mathrm{S}$ bond formation: scope and mechanistic studies. Chem. Eur. J. 23, $10522-10526$ (2017).

64. R. M. Denton, X. Tang, A. Przeslak, Catalysis of phosphorus (V)-mediated transformations: dichlorination reactions of epoxides under Appel conditions. Org. Lett. 12, 4678-4681 (2010). 
65. A. J. Cresswell, S. T. C. Eey, S. E. Denmark, Catalytic, stereospecific syn-dichlorination of alkenes. Nat. Chem. 7, 146-152 (2015).

66. J. C. Sarie, J. Neufeld, C. G. Daniliuc, R. Gilmour, Catalytic vicinal dichlorination of unactivated alkenes. ACS Catal. 9, 7232-7237 (2019).

67. H. Egami, T. Yoneda, M. Uku, T. Ide, Y. Kawato, Y. Hamashima, Difunctionalization of alkenes using 1-chloro-1,2-benziodoxol-3-(1H)-one. J. Org. Chem. 81, 4020-4030 (2016).

68. V. Wedek, R. Van Lommel, C. G. Daniliuc, F. De Proft, U. Hennecke, Organocatalytic, enantioselective dichlorination of unfunctionalized Alkenes. Angew. Chem. Int. Ed. 58, 9239-9243 (2019).

69. E. W. Tan, B. Chan, A. G. Blackman, A polar effects controlled enantioselective 1, 2chlorine atom migration via a chlorine-bridged radical intermediate. J. Am. Chem. Soc. 124, 2078-2079 (2002).

70. J. Wei, S. Liang, L. Jiang, Y. Mumtaz, W. B. Yi, Regioselective Chlorothiolation of Alkenes with Sulfonyl Chlorides. J. Org. Chem. 85, 977-984 (2019).

71. L. Benati, L. Capella, P. C. Montevecchi, P. Spagnolo, Reaction of 4'nitrobenzenesulfenanilide (NBSA) with Lewis acids. A study of its application in sulfenocyclization of alkenes and alkynes. Tetrahedron 50, 12395-12406 (1994).

72. P. Sanllehí, et al., The first fluorogenic sensor for sphingosine-1-phosphate lyase activity in intact cells. Chem. Commun. 53, 5441-5444 (2017).

73. J. M. Lopchuk, et al., Strain-release heteroatom functionalization: development, scope, and stereospecificity. J. Am. Chem. Soc. 139, 3209-3226 (2017).

74. K. Yuan, J. F. Soulé, V. Dorcet, H. Doucet, Palladium-Catalyzed Cascade $\mathrm{sp}^{2} \mathrm{C}-\mathrm{H}$ Bond Functionalizations Allowing One-Pot Access to 4-Aryl-1,2,3,4-tetrahydroquinolines from $N$-Allyl- $N$-arylsulfonamides. ACS Catal. 6, 8121-8126 (2016). 
75. J. C. Siu, J. B. Parry, S. Lin, Aminoxyl-catalyzed electrochemical diazidation of alkenes mediated by a metastable charge-transfer complex, J. Am. Chem. Soc. 141, 2825-2831 (2019).

76. R. L. Nyland II, Y. Xiao, P. Liu, C. L. Freel Meyers, IspG converts an epoxide substrate analogue to (E)-4-hydroxy-3-methylbut-2-enyl diphosphate: implications for IspG catalysis in isoprenoid biosynthesis. J. Am. Chem. Soc. 131, 17734-17735 (2009).

77. K. Yasui, K. Fugami, S. Tanaka, Y. Tamaru, Unsymmetrical ketone synthesis via a threecomponent connection reaction of organozincs, allylating agents, and carbon monoxide. $J$. Org. Chem. 60, 1365-1380 (1995).

78. D. Song, S. Cho, Y. Han, Y. You, W. Nam, Ratiometric fluorescent probes for detection of intracellular singlet oxygen. Org. Lett. 15, 3582-3585 (2013).

79. Y. Y. Ren, X. Zheng, X. Zhang, Bromotrifluoromethane: A Useful Reagent for Hydrotrifluoromethylation of Alkenes and Alkynes. Synlett 29, 1028-1032 (2018).

We thank M. Zesiger, the NMR service, the Molecular and Biomolecular Analysis Service (MoBiAS) and ETH Zürich for technical assistance. Support by SusInnoScience (JGU Mainz) is highly acknowledged. We thank S. Makai for assistance with GC-MS headspace analysis and helpful discussions, and E. Falk for reproducing one of the Lindane-soil experiments. S. Makai, E.

Falk, B. Bhawal, and T. Delcaillau are acknowledged for sharing chemicals. We thank the whole Morandi group for critical proof-reading of this manuscript. Funding: This project received funding from the European Research Council under the European Union's Horizon 2020 research and innovation program (Shuttle Cat, Project ID: 757608) and the ETH Zürich. X.D. acknowledges the Marie Skłodowska-Curie Action (HaloCat, Project ID: 886102) for a postdoctoral fellowship. 
J.L.R. is a recipient of a DFG fellowship through the Excellence Initiative by the Graduate School Materials Science in Mainz (GSC 266). Author contributions: B.M. and X.D. conceived the project. X.D. and J.L.R. designed and performed all the synthetic studies. S.W. and B.M. supervised the research. All authors contributed to the writing and editing of the manuscript. Competing interests: Authors declare no competing interests. Data and materials availability: All experimental data are available in the main text or the supplementary materials.

\section{Supplementary Materials:}

Materials and Methods

Figures S1-S41

10

Tables S1-S16

NMR Spectra

References (47-79) 